\title{
Desempeño productivo, consumo y digestibilidad de terneras lactantes criadas en pastoreo suplementadas con diferentes niveles de proteína bruta
}

\author{
Cardenas, J.E.G. ${ }^{1} ;$ Paulino, M.F. ${ }^{1}$; Lopes, S.A. ${ }^{\text {; }}$ Silva, A.G. ${ }^{\text {; }}$ Barros, L. ${ }^{2}$ y Valente, É.E.L. ${ }^{3}$
}

'Departamento de Zootecnia. Universidade Federal de Viçosa. Viçosa. Brasil.

2Departamento de Zootecnia e Extensão Rural. Universidade Federal de Mato Grosso. Cuiabá. Brasil.

${ }^{3}$ Departamento de Zootecnia. Universidade Estadual do Oeste do Paraná. Marechal Cândido Rondon. Brasil.

\section{PaLABRAS CLAVE ADICIONALES}

Creep-feeding.

Suplementación.

Urochloa decumbens.

\section{ADDITIONAL KEYWORDS}

Creep-feeding.

Supplementation.

Urochloa decumbens.

\section{INFORMACIÓN}

Cronología del artículo.

Recibido/Received: 12.9.2013

Aceptado/Accepted: 13.5.2015

On-line: 10.6 .2015

Correspondencia a los autores/Contact e-mail:

yojavier1@hotmail.com; javier.cardenas@ufv.br

\section{RESUMEN}

Impulsar el crecimiento acelerado de terneras a través de una ganancia de peso eficiente viene siendo el desafío para todo productor. El presente trabajo tuvo como objetivo evaluar el desempeño productivo, consumo y digestibilidad de terneras Nelore lactantes pastoreando Urochloa decumbens, suplementadas con niveles crecientes de proteína bruta (PB) durante la época de transición de lluvias y seca. Fueron utilizadas 60 terneras con edad media de $126 \pm 3,08$ días y peso medio inicial de $123 \pm 3,09 \mathrm{~kg}$, recibiendo $0,5 \mathrm{~kg} /$ animal durante 140 días. Los tratamientos consistieron en; 7,$3 ; 16,7 ; 25,6 ; 34,6$ y $43,2 \%$ de PB en base de la materia seca. Además, un tratamiento control que recibía apenas mixtura mineral ad libitum. Durante el período experimental se obtuvo una oferta de $90 \mathrm{~g}$ de materia seca potencialmente digestible (MSpd) por kilogramo de peso vivo, cantidad necesaria para conseguir desempeños satisfactorios, considerando que la recomendación es de 40 a $50 \mathrm{~g}$ de MSpd/ $\mathrm{kg}$. El promedio de la ganancia diaria de peso difirió $(p \leq 0,10)$ entre los grupos experimentales $(676 \mathrm{~g})$ y el grupo control $(619 \mathrm{~g})$. Las terneras suplementadas presentaron mayor consumo de $P B$, extracto etéreo, carbohidratos no fibrosos y nutrientes digestibles totales $(p \leq 0,10)$ y no se encontró diferencia en el consumo de materia seca total y materia seca de la leche. El flujo de compuestos nitrogenados microbianos en relación al nitrógeno ingerido presento perfil linear negativo, revelando que la producción microbiana diaria no fue influenciada por los tratamientos $(p>0,10)$. Se concluye que la suplementación es necesaria para incrementar la ganancia diaria de peso de terneras lactantes en pastoreo, sin embargo, la adición de diferentes niveles de PB en la dieta no difiere en el desempeño de estos animales cuando fueron suplementadas con $0,5 \mathrm{~kg} /$ día.

Performance productive, intake and digestibility of nursing calves raised on pasture supplemented with different levels of crude protein

\section{SUMMARY}

To promote the fast growth of calves through an efficient weight gain has been the challenge for any producer. This study aimed to evaluate the effects of increasing levels of cru protein (CP) on performance, intake, and digestibility of nursing Nellore calves, grazing Urochloa decumbens, during transition time from wet to dry season. 60 calves were used with age of $126 \pm 3.08$ days and average initial body weight of $123 \pm 3.09 \mathrm{~kg}$, receiving $0.5 \mathrm{~kg}$ per animal during 140 days. The treatments consisted of; $7.3,16.7,25.6,34.6$, and $43.2 \%$ $\mathrm{CP}$ based on dry matter basis. In addition, a control treatment was performed which animals had free choice access to mineral mixture. During the experimental period, $90 \mathrm{~g}$ of potentially digestible dry matter (PDDM) intake was offered whichthe amount necessary to achieve satisfactory performance obtained per kilogram of body weight, whereas the recommendation is $40-50 \mathrm{~g}$ of PDDM $/ \mathrm{kg}$. The average daily weight gain differed $(\mathrm{p} \leq 0.10)$ between experimental groups $(676 \mathrm{~g})$ and the control group $(619 \mathrm{~g})$. The supplemented calves had greater intake of $C P$, either extract, non-fibrous carbohydrates, and total digestible nutrients $(p \leq 0.10)$ and no difference was found for total dry matter intake and dry matter intake of milk. The flow of microbial nitrogen compounds in relationship to the ingested nitrogen presented a negative linear profile, revealing that daily microbial protein synthesis was not influenced by treatments $(p>0.10)$. Therefore, we concluded that supplementation is necessary to increase the daily weight gain of grazing nursing calves; however, the addition of different levels of $C P$ in the diet does not differ on performance when these animals were supplemented with $0.5 \mathrm{~kg} / \mathrm{d}$.

\section{INTRODUCCIÓN}

Con el pasar de los años se ha demostrado la importancia de crear modelos de manejo adecuados para la cría de bovinos a pasto, con el fin de maximizar su desarrollo y así garantizar un animal saludable, prepa- rado para los desafíos que traen las fases subsecuentes a la cría.

La leche es la principal fuente de nutrientes para los terneros, sin embargo, a medida que el animal crece, las necesidades nutricionales aumentan y la producción 
de leche disminuye. En los sistemas de cría de bovinos de carne, la leche producida por la vaca no es suficiente para garantizar las necesidades nutricionales de su cría a partir de los tres meses de edad (Henriques et al., 2011). Entre los nutrientes limitantes, los compuestos nitrogenados asumen mayor importancia durante el periodo de cría y la época seca del año, donde los bajos niveles de proteína en los pastos limitan la actividad de los microorganismos ruminales, afectando la digestibilidad y el consumo de forrajes, causando bajo desempeño en el animal (Detmann et al., 2004). Esta demanda por nutrientes se intensifica cuando buscamos reducir el ciclo productivo del animal para 14 o 16 meses de edad a la primera concepción.

Considerando que los pastos son la base de la alimentación de los bovinos en los ambientes tropicales se debe tener en cuenta los avances conceptuales sobre el manejo de las forrajeras. La materia seca potencialmente digestible (MSpd), integra calidad y cantidad en las pasturas independientemente de la época del año (Paulino et al., 2004), garantizando así una mayor extracción de nutrientes exigidos por el animal. Junto al pastoreo, la suplementación proteica es una estrategia que viene siendo implementada, permitiendo que los animales obtengan más energía por cada unidad de forraje consumida (Brito y Sampaio, 2001), mejorando la ingestión y aumentando el provecho de los nutrientes (Sampaio et al., 2010), la práctica de suplementación en bovinos de carne es capaz de garantizar la estabilidad en la producción animal (Fernandes et al., 2012).

Frente a esta situación, el presente trabajo tuvo como objetivo evaluar el desempeño productivo, consumo y digestibilidad de terneras Nelore lactantes pastoreando Urochloa decumbens, suplementadas con niveles crecientes de proteína bruta $(\mathrm{PB})$ durante la época de transición de lluvias-seca.

\section{MATERIAL Y MÉTODOS}

\section{LOCALIZACIÓN}

El estudio se realizó en el sector de Bovinocultura de Carne del Departamento de Zootecnia de la Universidad Federal de Viçosa, situado en el municipio de Viçosa, Minas Gerais, Brasil. Ubicada a una altura de 670 m.s.n.m., con una precipitación anual media de $1.300 \mathrm{~mm}$. La investigación fue realizada durante la fase de transición entre las épocas de lluvias y estación seca, de febrero a junio de 2011. Las variables climáticas durante el periodo experimental están representadas en la figura 1.

\section{MANEJO DE LOS ANIMALES}

Fueron utilizadas 60 terneras con por lo menos $50 \%$ de sangre Nelore lactantes, con edades media de $126 \pm 3,08$ días, peso medio inicial de $123 \pm 3,09 \mathrm{~kg}$, debidamente desparasitadas. Los animales fueron ubicados en seis potreros de aproximadamente siete hectáreas cada uno, cubiertos uniformemente con pasto Urochloa decumbens, dotados de bebederos y comederos, siendo estos últimos sombreados con tejas de asbesto y con entrada solo para las terneras, en formato creep-feeding. Los animales fueron rotados en los potreros cada siete días para evitar diferencias entre las áreas de pastoreo.

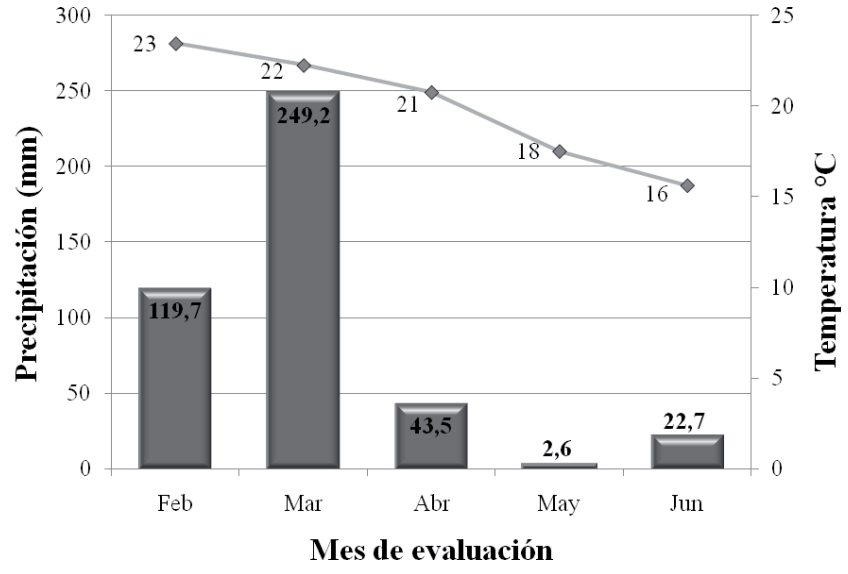

$\rightleftharpoons$ Precipitación $(\mathrm{mm}) \quad \prec$ Temperatura ${ }^{\circ} \mathrm{C}$

Figura 1. Precipitación en milímetros (mm) y temperatura media en ${ }^{\circ} \mathrm{C}$ durante el periodo experimental (Rainfall in millimeters $(\mathrm{mm})$ and average temperature in ${ }^{\circ} \mathrm{C}$ during experimental period). (Fuente: Departamento de Ingeniería Agrícola - UFV).

\section{DISEÑO EXPERIMENTAL}

El experimento tuvo una duración de 140 días, dividido en cinco periodos experimentales de 28 días cada uno. El delineamiento experimental fue enteramente casualizado con seis tratamientos y diez animales por tratamiento, constituidos por el aumento progresivo del porcentaje de proteína bruta 7,3; 16,7; 25,6; 34,6 y $43,2 \%$ con base en la materia seca del suplemento, además un tratamiento control (MM) que recibía apenas una mezcla mineral ad libitum (tablas I y II). Los suplementos fueron suministrados diariamente a las 10:00 am, $500 \mathrm{~g} /$ ternera. Sus madres recibían mezcla mineral ad libitum y $100 \mathrm{~g}$ de maíz molido para estimular la permanencia de estas cerca al comedero y así garantizar un mayor consumo por parte de las terneras.

La ganancia media diaria de peso (GMD) fue obtenida por la diferencia entre el peso corporal final e inicial, dividido por el número de días experimentales (140 días).

\section{MUESTREO Y ANÁLISIS FíSICO-QUÍMICO}

En el día $1^{\circ}$ y $14^{\circ}$ de cada periodo experimental, fueron realizadas las colectas de pastos por medio de simulación manual del pastoreo con el fin de evaluar las características cualitativas de los pastos.

Tabla I. Composición porcentual de los suplementos, con base en la materia natural (Composition of supplements (\%) on natural matter basis).

\begin{tabular}{lcccccc}
\hline Composición & \multicolumn{5}{c}{ Tratamientos } \\
\cline { 2 - 7 } & MM & 7,3 & 16,7 & 25,7 & 34,6 & 43,2 \\
Grano de maíz molido & 0 & 94 & 71 & 49 & 27 & 6 \\
Harina de soja $^{\text {Mezcla mineral }}{ }^{1}$ & 0 & 0 & 23 & 45 & 67 & 88 \\
& 100 & 6 & 6 & 6 & 6 & 6
\end{tabular}

${ }^{1}$ Composición porcentual: fosfato bicálcico, 50,00; cloruro de sodio, 47,15; sulfato de zinc, 1,50; sulfato de cobre, 0,75 ; sulfato de cobalto, 0,05; yodato de potasio, 0,05 y sulfato de magnesio: 0,5 . 
Tabla II. Composición químico bromatológica de los suplementos y del pasto (Chemical composition of supplements and pasture).

\begin{tabular}{lrrrrrl}
\hline Composición & \multicolumn{5}{c}{ Tratamientos } & U. decumbens * \\
\cline { 2 - 6 } & \multicolumn{1}{c}{16,3} & 25,7 & 34,6 & 43,2 & \\
\hline MS & 92,51 & 92,69 & 92,86 & 93,04 & 93,20 & $32,49 \pm 1,83$ \\
MO & 93,49 & 92,27 & 91,12 & 89,96 & 88,85 & $92,43 \pm 0,2$ \\
PB & 7,27 & 16,65 & 25,63 & 34,60 & 43,17 & $7,12 \pm 0,2$ \\
EE & 3,32 & 2,95 & 2,59 & 2,23 & 1,89 & $1,75 \pm 0,02$ \\
FDNcp & 9,42 & 8,63 & 7,87 & 7,11 & 6,38 & $64,03 \pm 0,4$ \\
CNF & 73,47 & 64,05 & 55,03 & 46,01 & 37,41 & $19,53 \pm 0,3$ \\
FDNi & 0,96 & 0,89 & 0,83 & 0,77 & 0,71 & $19,95 \pm 0,2$ \\
NIDN & 10,52 & 11,85 & 13,11 & 14,38 & 15,59 & $17,67 \pm 1,02$ \\
FDAcp & 4,54 & 5,53 & 6,47 & 7,41 & 8,31 & $37,08 \pm 1,63$ \\
\hline
\end{tabular}

$\mathrm{MS}=$ materia seca; $\mathrm{MO}=$ materia orgánica; $\mathrm{PB}=$ proteína bruta; $\mathrm{EE}=$ extracto etéreo; FDNcp= fibra en detergente neutro corregida para cenizas y proteína; $\mathrm{CNF}=$ carbohidratos no-fibrosos; $\mathrm{FDNi}=\mathrm{fi}$ bra en detergente neutro indigestible; NIDN= nitrógeno insoluble en detergente neutro; FDAcp= fibra en detergente ácido corregida para cenizas y proteína; *medias \pm desviación típica.

Las muestras fueron pre-secadas en estufa a $60^{\circ} \mathrm{C}$ con ventilación forzada durante 72 horas y posteriormente molidas (molino tipo Thomas Wiley, a 1 y $2 \mathrm{~mm}$ ), junto a estas muestras, los ingredientes utilizados en los suplementos también fueron cuantificados. Las proporciones de materia seca (MS, índice $\left.n^{\circ} 920,39\right)$, proteína bruta (PB, índice $\left.\mathrm{n}^{\circ} 954,01\right)$, y extracto etéreo (EE, índice $\left.n^{\circ} 920,39\right)$ fueron analizadas según la metodología descrita por la AOAC (1999); fibra en detergente neutro (FDN) por medio de las recomendaciones de Mertens (2002), se utilizo $\alpha$-amilasa termoestable y se omitió el uso de sulfito de sodio; fibra en detergente acido (FDA) según la técnica secuencial descrita por Van Soest y Robertson (1985), las correcciones para proteína y cenizas en la FDNcp y FDAcp siguieron los procedimientos descritos por Licitra et al. (1996) y Mertens (2002), respectivamente; fibra en detergente neutro indigestible (FDNi), fue adquirida después de la incubación en sacos F57 (Ankom ${ }^{\circledR}$ ) in situ por 288 horas, de acuerdo con Valente et al. (2011); nitrógeno insoluble en detergente neutro (NIDN) siguiendo lo descrito por Licitra et al. (1996).

La cuantificación de los carbohidratos no fibrosos (CNF) se realizó según la metodología de Detmann y Valadares Filho (2010):

$$
\mathrm{CNF}=100-(\% \mathrm{~PB}+\% \mathrm{FDNcp}+\% \mathrm{EE}+\% \mathrm{M})
$$

Simultáneamente, en el $14^{\circ}$ día de cada periodo experimental fueron realizadas colectas de pasto, esta vez, por medio de un corte arras del suelo en cuatro áreas delimitadas por un cuadrado metálico de 0,5 x 0,5 metros, seleccionados aleatoriamente en cada potrero experimental, con el propósito de evaluar la disponibilidad de materia seca total (MST) y la materia seca potencialmente digestible (MSpd) por hectárea. Estas muestras también fueron pesadas y llevadas inmediatamente a estufa a $60^{\circ} \mathrm{C}$ por 72 horas, molidas y luego sometidas a análisis para definir las proporciones de MS, FDN y FDNi.
La MSpd fue estimada según la fórmula descrita por Paulino et al. (2006):

$$
\mathrm{MSpd}=0,98 \times(100-\mathrm{FDN})+(\mathrm{FDN}-\mathrm{FDNi})
$$

Para estimar el consumo de leche, fueron realizadas colectas totales de leche a los 35, 82 y 115 días. La leche fue pesada para estimar la producción y posteriormente fueron realizados los análisis de proteína y grasa.

Para la evaluación de las características nutricionales fue realizado en el día 70, un ensayo de consumo y digestibilidad con duración de nueve días, de los cuales seis fueron destinados a la adaptación de los indicadores. Para estimar la excreción fecal, fue utilizado como indicador externo el óxido crómico $\left(\mathrm{Cr}_{2} \mathrm{O}_{3}\right)$, para estimar el consumo individual de suplemento, fue suministrado el dióxido de titanio $\left(\mathrm{TiO}_{2}\right)$ y para evaluar el consumo de MST y MS de pasto fue utilizado como indicador interno FDNi. En los últimos tres días fueron realizadas colectas de heces a las 7:00, 11:00 y 15:00 horas, inmediatamente después de la defecación o directamente en el recto de los animales, colectando aproximadamente $300 \mathrm{~g}$ de materia fecal. Las muestras se secaron en estufa con circulación forzada de aire a $60^{\circ} \mathrm{C}$ durante 72 horas, posteriormente fueron molidas y destinadas para analizar los porcentajes de cromo por espectrofotometría de absorción atómica (Willians et al., 1962), dióxido de titanio por colorimetría (Titgemeyer et al., 2001) y de MS, PB, EE, FDNcp y FDNi.

La excreción de materia seca fecal fue estimada utilizando el indicador óxido crómico, siendo estimada en base a la razón entre la cantidad de indicador suministrado y la concentración del indicador en las heces (IF).

Excreción de heces $(\mathrm{g} /$ día $)=\frac{\text { Indicador suministrado }(\mathrm{g})}{\operatorname{IF}(\%)} \times 100$

La estimativa del consumo individual de suplemento (CISup) fue conseguida a través de la siguiente ecuación:

$$
\text { CISup }(g / \text { día })=\frac{E F \times C I F i}{I F G} \times \text { SupFG }
$$

Dónde:

CISup = consumo individual de suplemento (g/día);

$\mathrm{EF}=$ excreción fecal (g/día);

$\mathrm{CIFi}=$ concentración del indicador en las heces del animal $(\mathrm{kg} / \mathrm{kg})$;

IFG= indicador presente en el suplemento suministrado a el grupo (g/día);

SupFG= cantidad de suplemento suministrado a el grupo de animales (g/día).

La estimación del consumo individual de materia seca de pasto (CIMSP) fue realizada utilizando como indicador interno la FDNi, según Detmann et al. (2001), utilizando la siguiente ecuación:

$\operatorname{CIMSP}(\mathrm{g} /$ día $)=\frac{[(E F \times \text { FDNiFE })-\text { CMSS } x \text { FDNiSup }]}{\text { FDNiPasto }}$ 
Dónde:

CIMSP = consumo individual de materia seca de pasto (g/día);

$\mathrm{EF}=$ excreción fecal (kg/día);

FDNiFE= concentración de FDNi fecal $(\mathrm{kg} / \mathrm{kg})$;

$\mathrm{CMSS}=$ consumo de materia seca de suplemento (kg/día);

FDNiSup= concentración de FDNi en el suplemento $(\mathrm{kg} / \mathrm{kg}) \mathrm{y}$

FDNiPasto $=$ concentración de FDNi en el forraje ( $\mathrm{kg} / \mathrm{kg})$.

En el último día de ensayo, fue realizada, una colecta spot de muestras de orina $(10 \mathrm{ml})$, por micción espontanea, cuatro horas después de suministrado el suplemento para evaluar los parámetros nutricionales. Las muestras de orina fueron diluidas en $40 \mathrm{ml}$ de ácido sulfúrico $\left(\mathrm{H}_{2} \mathrm{SO}_{4}-0,036 \mathrm{~N}\right)$ y congeladas a $-20^{\circ} \mathrm{C}$. En el mismo día, fue realizada una toma de sangre, por punción de la vena yugular, usando tubos de vacío y gel separador (BD Vacuntainer® SST II Advance), las muestras fueron llevadas al laboratorio, centrifugadas a $2500 \times \mathrm{G}$ durante 20 minutos y el suero resultante fue congelado a $-20^{\circ} \mathrm{C}$.

Para estimar los porcentajes urinarios de urea, fue utilizada la prueba enzimático-colorimétrica (InVitro ${ }^{\circledR}$, Uréia Liquicolor), para ácido úrico la prueba enzimático-colorimétrica con factor aclarante de lípidos (InVitro®, Uric Acid Liquicolor) y los porcentajes de creatinina fueron estimados por el método de Jaffé modificado (InVitro $\AA$, Creatinina).

Los análisis de alantoína fueron realizados por el método colorimétrico, conforme al descrito por Chen y Gomes (1992). La excreción total de derivados de purinas fue calculada por la suma de las cantidades de alantoína y ácido úrico excretado en la orina, expresadas en $\mathrm{mmol} /$ día.

El volumen urinario diario fue estimado por la relación entre la excreción diaria de creatinina (EC), adoptando la ecuación propuesta por Costa y Silva et al. (2012):

$$
\mathrm{EC}(\mathrm{g} / \text { día })=0,0345 \times \mathrm{PCJ}^{0,9491}
$$

Dónde:

PCJ= peso corporal en ayuno.

De esta forma, La excreción urinaria diaria de compuestos nitrogenados fue el producto entre su concentración en las muestras spot y el valor estimado del volumen urinario.

Las purinas absorbidas fueron calculadas a partir de la excreción de derivados de purinas, mediante la ecuación (Barbosa et al., 2011):

Dónde:

$$
\mathrm{PA}=\frac{\mathrm{DP}-0,301 \times \mathrm{PC}^{0,75}}{0,80}
$$

$\mathrm{PA}=$ purinas absorbidas (mmol/día);
$\mathrm{DP}=$ excreción de derivados de purinas (mmol/ día);

0,301= excreción endógena de derivados de purina en la orina ( $\mathrm{mmol}$ ) por unidad de peso metabólico $\left(\mathrm{PC}^{0,75}\right) \mathrm{y}$

$0,80=$ recuperación de purinas absorbidas como derivados de purina en la orina.

La síntesis ruminal de compuestos nitrogenados microbianos fue estimada en función de las PA utilizando la ecuación descrita por Chen y Gomes (1992), con excepción de la relación $\mathrm{N}$ de purinas: $\mathrm{N}$ total de las bacterias de 0,134 conforme Valadares et al. (1999):

$$
\mathrm{Nmic}=\frac{(70 \times \mathrm{PA})}{(0,83 \times 0,134 \times 1000)}
$$

Dónde:

Nmic $=$ flujo de compuestos nitrogenados microbianos (g/día);

$70=$ cantidad de $\mathrm{N}$ de purinas ( $\mathrm{mg} \mathrm{N} / \mathrm{mol})$;

0,83= digestibilidade de las purinas bacterianas y

$0,134=$ relación $\mathrm{N}$ purinas: $\mathrm{N}$ total en las bacterias.

\section{ANÁLISIS ESTADÍSTICO}

Para los análisis estadísticos se utilizó el PROC GLM del SAS (versión 9.1). Adoptándose un nivel de $10 \%$ de probabilidad para el error tipo I. Las comparaciones entre las medias observadas fueron realizadas por medio de la descomposición de la suma de los cuadrados para los tratamientos en contraste ortogonales relativos a comparación entre suplementados y no suplementado. Al igual se evaluó el efecto lineal, cuadrático y cubico en los distintos niveles de PB.

\section{RESULTADOS Y DISCUSIÓN}

Los niveles de productividad de los bovinos criados en pastoreo varían conforme al tipo de forraje, donde la calidad se da en función de la fertilidad de los suelos, de los factores climáticos y del manejo. La interpretación del forraje disponible al pastoreo como recurso nutricional basal, debe ser conducida sobre la óptica de la fracción potencialmente transformable en producto animal, la cual se puede conseguir aplicando el concepto de MSpd, ya que esta integra cantidad y calidad independientemente de la época del año (Paulino et al., 2004).

La disponibilidad media de materia seca total (MST) y de MSpd del pasto Urochloa decumbens durante el periodo experimental fue de 3,82 y 2,15 t/ ha, respectivamente (figura 2). Durante el periodo de 28 días la oferta fue de $15.106 \mathrm{~kg}$ por potrero, es decir, $90 \mathrm{~g}$ de MSpd por kilogramo de peso vivo, cantidad necesaria para conseguir desempeños satisfactorios, considerando que la recomendación es de 40 a $50 \mathrm{~g}$ de MSpd/kg de peso vivo, como fue descrito por Paulino et al. (2004). Esto es representativo cuando consideramos por ejemplo, 10 grupos conformados cada uno por una vaca y una ternera, de aproximadamente 600 


\section{DisponibilidAd DE ForRAJE}

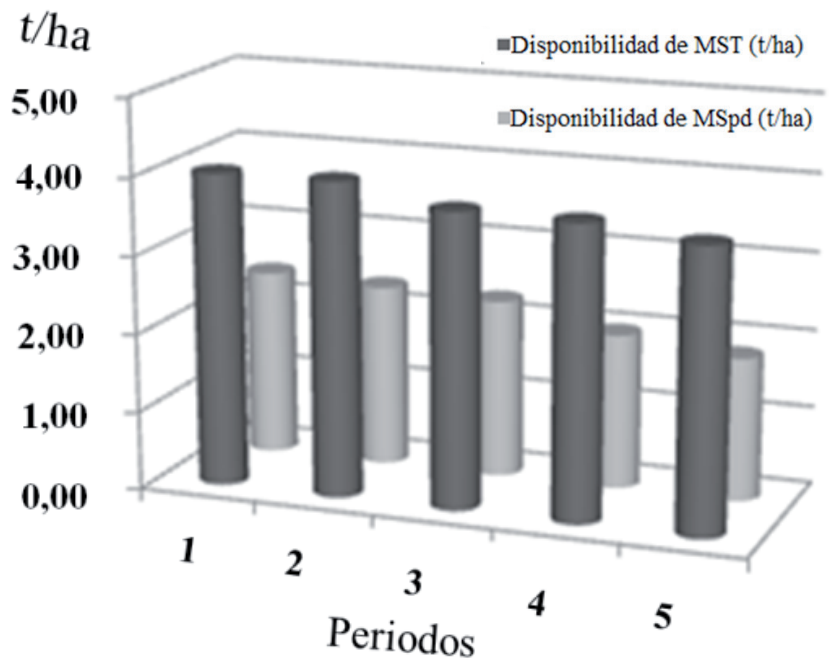

Figura 2. Disponibilidad de materia seca total (MST) y de materia seca potencialmente digestible (MSpd) en el pasto (Total dry matter availability (MST) and potentially digestible dry matter (MSpd) of pasture used in the experiment).

$\mathrm{kg}$ de peso vivo, en 28 días necesitarían $8400 \mathrm{~kg}$ de MSpd, este valor indica que en este experimento la cantidad de pasto no fue una limitante para mejorar el desempeño de los animales. Por lo cual, es posible afirmar que fueron proporcionadas condiciones adecuadas para que los animales presentaran el consumo de forraje maximizado, lo que explica la ausencia de respuesta en el consumo de MST por las terneras que recibían suplementación con distintos niveles de PB en relación a las del grupo control. Se observó un mayor consumo de forraje en $\mathrm{g} / \mathrm{kg}$ de PC para los animales del tratamiento control, lo que puede ser explicado por el efecto substitutivo del consumo de pasto por el no consumo de suplemento (tabla III). Además, el forraje Urochloa decumbens colectada por simulación manual del pastoreo, presento cantidades de 71,2 g de $\mathrm{PB} / \mathrm{kg}$ de MS (tabla II), estando dentro de los valores mínimos de $7-8 \%$ de PB en la dieta basal propuestos por Lazzarini et al. (2009) como necesarios para que se dé una adecuada utilización de la fibra en detergente neutro (FDN) de la forrajera consumida, que sería la principal fuente nutricional para los animales criados en praderas. Sin embargo, este valor está por debajo de los 10\% de PB citado por Sampaio et al. (2009) como cantidad ideal para la optimización en la utilización del substrato energético de la pastura, lo que justifica la suplementación de compuestos nitrogenados y así garantizar la máxima extracción de los nutrientes presentes en el forraje y consecuentemente aumentar el desempeño de las terneras.

Los animales que consumieron suplementos presentaron mayor consumo de proteína bruta (PBT), extracto etéreo (EET), carbohidratos no fibrosos $(\mathrm{CNF})$ y

Tabla III. Medias, coeficiente de variación $(\mathrm{CV})$ e indicativo de significación para el consumo de los componentes de la dieta por las terneras suplementadas y no suplementadas (Means, coefficient of variation (CV) and significance for nutrient intake of calves receiving different supplements).

\begin{tabular}{|c|c|c|c|c|c|c|c|c|c|c|c|}
\hline \multirow[t]{2}{*}{ Descripción } & \multicolumn{6}{|c|}{ Tratamientos ${ }^{1}$} & \multirow[t]{2}{*}{ CV (\%) } & \multicolumn{4}{|c|}{$(\text { Valor }-p)^{2}$} \\
\hline & $\mathrm{MM}$ & 7,3 & 16,7 & 25,7 & 34,6 & 43,2 & & $\mathrm{CO}$ & $\mathrm{L}$ & Q & C \\
\hline \multicolumn{12}{|l|}{ kg/día } \\
\hline MST & 3,003 & 3,192 & 3,314 & 3,163 & 3,432 & 3,412 & 18,89 & ns & ns & ns & ns \\
\hline MSP & 2,050 & 1,744 & 1,846 & 1,704 & 1,903 & 1,906 & 25,79 & ns & ns & ns & ns \\
\hline MSS & - & 0,462 & 0,463 & 0,464 & 0,465 & 0,465 & 44,30 & - & ns & ns & ns \\
\hline MSL & 0,951 & 0,949 & 0,970 & 0,961 & 1,000 & 1,006 & 21,66 & ns & ns & ns & ns \\
\hline PBT & 0,387 & 0,397 & 0,447 & 0,500 & 0,586 & 0,612 & 19,41 & + & + & ns & ns \\
\hline PBL & 0,241 & 0,232 & 0,255 & 0,266 & 0,254 & 0,245 & 19,29 & ns & ns & ns & ns \\
\hline EET & 0,343 & 0,381 & 0,376 & 0,360 & 0,399 & 0,371 & 16,18 & $*$ & ns & ns & ns \\
\hline EEL & 0,312 & 0,320 & 0,341 & 0,338 & 0,347 & 0,332 & 18,75 & ns & ns & ns & ns \\
\hline $\mathrm{CNF}$ & 0,745 & 1,032 & 1,058 & 1,028 & 1,091 & 1,083 & 10,75 & * & ns & ns & ns \\
\hline FDNcp & 1,321 & 1,176 & 1,245 & 1,143 & 1,214 & 1,239 & 25,72 & ns & ns & ns & ns \\
\hline FDNi & 0,413 & 0,370 & 0,369 & 0,346 & 0,370 & 0,376 & 25,54 & ns & ns & ns & ns \\
\hline FDND & 0,944 & 0,858 & 0,919 & 0,823 & 0,879 & 0,899 & 25,42 & ns & ns & ns & ns \\
\hline NDT & 2,450 & 2,676 & 2,756 & 2,684 & 2,983 & 2,960 & 13,58 & * & * & ns & ns \\
\hline CMSD & 2,120 & 2,287 & 2,347 & 2,243 & 2,514 & 2,473 & 18,92 & * & ns & ns & ns \\
\hline \multicolumn{12}{|c|}{ g/kg de peso corporal } \\
\hline MST & 17,997 & 18,005 & 19,258 & 17,761 & 19,096 & 18,385 & 17,21 & ns & ns & ns & ns \\
\hline MSP & 12,259 & 9,723 & 10,514 & 9,468 & 10,373 & 10,263 & 20,69 & * & ns & ns & ns \\
\hline FDNcp & 7,897 & 6,554 & 7,092 & 6,344 & 6,620 & 6,673 & 20,52 & * & ns & ns & ns \\
\hline FDNi & 2,468 & 2,063 & 2,107 & 1,922 & 2,020 & 2,028 & 23,32 & * & ns & ns & ns \\
\hline
\end{tabular}

${ }^{1}$ Medias ajustadas, para los consumos de materia seca total (MST), MS de pasto (MSP), MS de suplemento (MSS) y MS de la leche (MSL), proteína bruta total (PBT) y PB de la leche (PBL), extracto etéreo total (EET) y de la leche (EEL), fibra en detergente neutro corregida para cenizas y proteína (FDNcp), carbohidratos no fibrosos (CNF), FDN digestible (FDND), FDN indigestible (FDNi) y de nutrientes digestibles totales (NDT). ${ }^{2}$ Coeficientes de variación (CV) e indicativos de significación para efecto entre animales suplementados y no suplementados $(C O)$, así como de orden linear $(L)$, cuadrática $(Q)$ y cúbica $(C) .{ }^{*}=$ Significativo al nivel de 0,1 de probabilidad $(p<0,1) ;+=p<0,1 ; n s=$ no significativo $(p>0,1)$. 
Tabla IV. Medias, coeficiente de variación (CV) e indicativo de significación de la digestibilidad de los nutrientes para los diferentes alimentos (Means, coefficient of variation (CV) and significance for nutrient digestibility of calves receiving different supplements).

\begin{tabular}{|c|c|c|c|c|c|c|c|c|c|c|c|}
\hline \multirow[t]{2}{*}{ Descripción } & \multicolumn{6}{|c|}{ Tratamientos $^{1}$} & \multirow[t]{2}{*}{$\mathrm{CV}(\%)$} & \multicolumn{4}{|c|}{$(\text { Valor }-p)^{2}$} \\
\hline & MM & 7,3 & 16,7 & 25,7 & 34,6 & 43,2 & & $\mathrm{CO}$ & $\mathrm{L}$ & Q & C \\
\hline \multicolumn{12}{|l|}{ kg/día } \\
\hline DMS & 0,706 & 0,717 & 0,708 & 0,709 & 0,734 & 0,724 & 5,21 & ns & ns & ns & ns \\
\hline DMO & 0,740 & 0,750 & 0,743 & 0,744 & 0,763 & 0,752 & 4,46 & $\mathrm{~ns}$ & ns & ns & ns \\
\hline DPB & 0,693 & 0,708 & 0,719 & 0,734 & 0,789 & 0,776 & 6,62 & + & + & ns & $\mathrm{ns}$ \\
\hline DEE & 0,897 & 0,915 & 0,896 & 0,897 & 0,891 & 0,908 & 3,83 & $\mathrm{~ns}$ & ns & ns & ns \\
\hline DCNF & 0,719 & 0,735 & 0,720 & 0,749 & 0,769 & 0,758 & 7,35 & ns & * & ns & ns \\
\hline DFDN $_{\mathrm{CP}}$ & 0,715 & 0,728 & 0,743 & 0,718 & 0,727 & 0,725 & 3,89 & $\mathrm{~ns}$ & ns & ns & $\mathrm{ns}$ \\
\hline NDT & 0,818 & 0,857 & 0,843 & 0,853 & 0,879 & 0,875 & 9,33 & $\mathrm{~ns}$ & ns & ns & $\mathrm{ns}$ \\
\hline
\end{tabular}

${ }^{1}$ Medias ajustadas, para la digestibilidad aparente total (en $\mathrm{kg} / \mathrm{kg}$ ) de la materia seca (DMS) materia orgánica (DMO), proteína bruta (DPB), extracto etéreo (DEE) carbohidratos no-fibrosos (DCNF), fibra en detergente neutro corregida para cenizas y proteína (DFDNcp) y nutrientes digestivos totales (NDT) de la dieta consumida por cada grupo de terneras. ${ }^{2}$ Coeficientes de variación (CV) e indicativos de significación para efecto entre animales suplementados y no suplementados (CO), así como de orden linear (L), cuadrática (Q) y cúbica (C). * significativo al nivel de 0,1 de probabilidad $(p<0,1) ;+=p<0,1 ; n s=$ no significativo $(p>0,1)$.

Tabla V. Parámetros metabólicos y de excreción (Excretion and metabolic parameters).

\begin{tabular}{|c|c|c|c|c|c|c|c|c|c|c|c|}
\hline \multirow[t]{2}{*}{ Descripción } & \multicolumn{6}{|c|}{ Tratamientos $^{1}$} & \multirow[t]{2}{*}{ CV (\%) } & \multicolumn{4}{|c|}{$(\text { Valor }-p)^{2}$} \\
\hline & MM & 7,3 & 16,7 & 25,7 & 34,6 & 43,2 & & $\mathrm{CO}$ & L & Q & C \\
\hline \multicolumn{12}{|l|}{ kg/día } \\
\hline $\mathrm{U}: \mathrm{C}$ & 0,100 & 0,122 & 0,144 & 0,166 & 0,180 & 0,211 & 35,87 & 0.0009 & 0.0002 & 0.8519 & 0.7680 \\
\hline NUS & 11,900 & 10,600 & 13,297 & 13,489 & 13,435 & 15,699 & 18,44 & 0,0839 & $<, 0001$ & 0,6930 & 0,0483 \\
\hline NMIC & 36,624 & 38,564 & 38,023 & 40,074 & 42,475 & 42,635 & 32,65 & 0,1298 & 0,3452 & 0,4372 & 0,5392 \\
\hline EFM & 114,865 & 109,343 & 117,823 & 106,382 & 116,247 & 115,357 & 32,11 & 0,4007 & 0,8291 & 0,8541 & 0,6529 \\
\hline NMICR & 0,630 & 0,645 & 0,557 & 0,550 & 0,481 & 0,463 & 37,51 & 0,1971 & 0,0367 & 0,7489 & 0,8787 \\
\hline $\mathrm{DP}$ & 30,388 & 34,072 & 31,385 & 33,571 & 34,516 & 35,484 & 22,93 & 0,1825 & 0,4291 & 0,4976 & 0,5248 \\
\hline
\end{tabular}

${ }^{1}$ Medias ajustadas, sobre la excreción de nitrógeno uréico sérico (NUS, mg/dl), relación urea:creatinina (U:C), producción de compuestos nitrogenados microbianos (NMIC, g/día), eficiencia microbiana (EFM, g/kg), compuestos nitrogenados microbianos y derivados de purinas (DP, mmol/día). ${ }^{2}$ Coeficientes de variación (CV) e indicativos de significancia para efecto de los suplementos.

nutrientes digestibles totales (NDT), en relación a los animales del grupo control ( $\mathrm{p}<0,10$; tabla III). Esto se le atribuye al mayor aporte de los constituyentes en el suplemento (tabla II), una vez que no hubo diferencias en el consumo de materia seca de la leche, principal fuente de estos componentes. Además, la suplementación con PB presentó un efecto lineal creciente $(p<0,10)$, el principal factor de esta diferencia fue la proteína oriunda del suplemento, ya que el consumo de PB de la leche no varió entre los grupos evaluados, por lo cual no contribuye para las diferencias observadas en el consumo de PB (tablas II y III).

El consumo de NDT fue ampliado por el suministro del suplemento, presentando un efecto lineal creciente $(p<0,10)$. La mayor concentración de energía en el suplemento en relación al pasto llevo a mayor ingestión de NDT, habiendo consumido la misma cantidad de materia seca total (MST).

Los animales del grupo control presentaron mayor consumo de FDNcp, y de FDNi expresado en $\mathrm{g} / \mathrm{kg}$ PC ( $<<0,10$; tabla III). La cantidad de forraje ofertado contribuye para que el grupo control presente un mayor consumo de materia seca de pasto (MSP) en
$12,3 \mathrm{~g} / \mathrm{kg}$ de $\mathrm{PC}$, en comparación a los animales que recibieron suplementación $10 \mathrm{~g} / \mathrm{kg}$ de PC, justificando la diferencia presente en el FDNcp, y FDNi viendo que la principal fuente de estas fracciones es el pasto.

La digestibilidad aparente total de la materia seca, materia orgánica, extracto etéreo, FDNcp y NDT para la dieta consumida por las terneras, no difirió ( $p>0,10$; tabla IV) entre los tratamientos. Esto puede ser atribuido a las condiciones de los pastos que proporcionaron una MSpd abundante durante todo el periodo experimental, lo que posibilita la selección de material de excelente calidad por los animales.

Se observó efecto de la suplementación y efecto lineal creciente para la digestibilidad de la $\mathrm{PB}(\mathrm{p}<0,10$; tabla IV), debido a la mayor ingestión de PB de mayor degradación en la dieta de los animales que recibieron suplemento, visto que el porcentaje de nitrógeno insoluble en detergente neutro para el suplemento fue menor que para la forraje (tabla II). Además del aumento progresivo de $\mathrm{PB} ; 7,3 ; 16,7 ; 25,6 ; 34,6$ y $43,2 \%$ en la dieta de los animales suplementados lo que puede haber aumentado la digestibilidad de la fracción proteica. 
Tabla VI. Resultados para el desempeño en terneras lactantes suplementadas a pasto (Performance of nursing calves raised on pasture).

\begin{tabular}{|c|c|c|c|c|c|c|c|c|c|c|c|}
\hline \multirow[t]{2}{*}{ Descripción } & \multicolumn{6}{|c|}{ Tratamientos $^{1}$} & \multirow[t]{2}{*}{$\mathrm{CV}(\%)$} & \multicolumn{4}{|c|}{$(\text { Valor }-p)^{2}$} \\
\hline & MM & 7,3 & 16,7 & 25,7 & 34,6 & 43,2 & & $\mathrm{CO}$ & $\mathrm{L}$ & $\mathrm{Q}$ & C \\
\hline \multicolumn{12}{|l|}{ kg/día } \\
\hline $\mathrm{PCl}$ & 120,5 & 120,3 & 119,2 & 121,1 & 124,9 & 130,3 & & - & - & - & - \\
\hline PCF & 207,9 & 218,2 & 216,3 & 213,3 & 217,5 & 228,1 & 14,37 & ns & ns & ns & ns \\
\hline GMD & 0,619 & 0,694 & 0,688 & 0,653 & 0,656 & 0,693 & 14,18 & + & ns & ns & ns \\
\hline
\end{tabular}

${ }^{1}$ Medias ajustadas, para el peso corporal inicial (PCI) en kg, peso corporal final (PCF) en kg, ganancia media diaria (GMD) en kg. ${ }^{2} \mathrm{Coeficientes}$ de variación (CV) e indicativos de significación para efecto entre animales suplementados y no suplementados (CO), así como de orden linear $(L)$, cuadrática $(Q)$ y cúbica $(C)$. * significativo al nivel de 0,1 de probabilidad $(p<0,1)$; $+=p<0,1$; ns= no significativo ( $p>0,1)$.

El efecto lineal creciente $(\mathrm{p}<0,10)$ observado en la digestibilidad aparente de los CNF pudo haber sido debido a una menor contribución de la fracción metabólica fecal en proporción a el consumo de suplemento.

La suplementación y efecto lineal creciente sobre la relación entre urea:creatinina y para el nitrógeno ureico sérico (U:C g/día; NUS, mg/dl; tabla V) fue significativo $(\mathrm{p}<0,10)$, debido al mayor consumo de PB de los animales suplementados, una vez que la concentración de NUS y urea esta positivamente correlacionada con la ingestión de nitrógeno. La urea es la forma de excreción del metabolismo de $\mathrm{N}$ por los mamíferos con gasto de energía, a medida que aumenta el nivel de suplementación ocurre un incremento de la pérdida de $\mathrm{N}$.

El NUS viene siendo frecuentemente utilizado con la finalidad de suministrar informaciones adicionales del estado nutricional proteico de rumiantes, incluyendo la respuesta de este a determinada dieta. El consumo de suplemento a partir de $430 \mathrm{~g} /$ día permite que los valores de NUS ultrapasen los niveles de 13,52 a $15,15 \mathrm{mg} / \mathrm{dl}$, indicando una probable perdida de proteína (Valadares et al. 1997), entre tanto, los animales del grupo control presentaron un valor de NUS muy próximo a este nivel $(11,90 \mathrm{mg} / \mathrm{dl})$, lo que puede indicar las condiciones nutricionales que permitieron que el grupo control presentara desempeño próximo de los grupos suplementados 10,$60 ; 13,29 ; 13,48 ; 13,43$ y 15,69 para 7,$3 ; 16,7 ; 25,6 ; 34,6$ y 43,2\% PB respectivamente.

No hubo efecto $(p>0,10)$ de los niveles de suplementación sobre el flujo de compuestos nitrogenados microbianos (NMIC-g/día), al igual que la eficiencia de síntesis microbiana (EFM) expresada como g PB$\mathrm{mic} / \mathrm{kg}$ de NDT consumido. Los tratamientos no influenciaron la producción microbiana diaria $(\mathrm{p}>0,10)$, posiblemente porque la cantidad de proteína ofrecida en los suplementos fue insuficiente para mejorar el crecimiento microbiano. Por lo tanto el flujo de compuestos nitrogenados microbianos en relación al nitrógeno ingerido (NMCIR) presento perfil lineal negativo (tabla V), debido a que el NMIC fue el mismo entre los tratamientos, pero el consumo de nitrógeno fue superior para los mayores niveles de PB.

En el presente trabajo fue encontrado un menor desempeño para los animales que consumieron solo mezcla mineral (grupo control). El promedio de la ganancia media diaria $(G M D)$ difirió $(p \leq 0,10)$ entre los grupos experimentales (676 g) y el grupo control (619 g). Sin embargo, no se presentó diferencia significativa entre los animales que consumieron diferentes niveles de PB (tabla VI), esto indica que las terneras ya habían atendido las exigencias de PB necesarias para este nivel de ganancia de peso, por lo tanto, la utilización del suplemento seria de orden económico de acuerdo a la respuesta de la suplementación energética. La GMD conseguida en este trabajo fue superior a los relatados por Nogueira et al. (2006) y Porto et al. (2009) que fue de 600 y $610 \mathrm{~g}$, respectivamente. Según Paulino et al. (2004), es necesario una GMD superior a $600 \mathrm{~g}$ para conseguir una primera concepción a los 14-15 meses de edad.

\section{CONCLUSIÓN}

La suplementación es necesaria para incrementar la ganancia diaria de peso de terneras lactantes en pastoreo, sin embargo, la adición de diferentes niveles de $\mathrm{PB}$ en la dieta no difiere en el desempeño de estos animales cuando fueron suplementadas con $0,5 \mathrm{~kg} /$ día.

\section{AGRADECIMIENTOS}

A la Fundación de Amparo a la Pesquisa del Estado de Minas Gerais (FAPEMIG), por la ayuda en la realización de este trabajo.

Al Departamento de Zootecnia de la Universidad Federal de Viçosa, por la contribución logística en la realización de este trabajo.

\section{BIBLIOGRAFÍA}

AOAC. 1995. Official methods of analysis. $16^{\text {th }}$ ed. Association of Official Analytical Chemists. Washington, D.C.

Barbosa, A.M.; Valadares, R.F.D.; Valadares Filho, S.C.; Pina, D.S.; Detmann, E. and Leão, M.I. 2011. Endogenous fraction and urinary recovery of purine derivatives obtained by different methods in Nellore cattle. J Anim Sci, 89: 510-519.

Brito, R.M. e Sampaio, A.A.M. 2001. Técnicas de suplementação de pastagens na criação de bezerros de corte: creep-feeding. Funep. Jaboticabal. 126 pp.

Chen, X.B. and Gomes, M.J. 1992. Estimation of microbial protein supply to sheep and cattle based on urinary excretion of purine derivatives. An overview of the technical details. (Occasional publication) Inter- 
national Feed Research Unit. Rowett Research Institute. Bucksburnd. Aberdeen. 21 pp.

Costa e Silva, L.F.; Valadares Filho, S.C.; Chizzotti, M.L.; Rotta, P.P. Prados, L.F.; Valadares, R.F.D.; Zanetti, D. and Braga, J.M.S. 2012. Creatinine excretion and relationship with body weight of Nellore cattle. Rev Bras Zootecn, 41: 807-810.

Detmann, E.; Paulino, M.F.; Zervoudakis, J.T.; Valadares Filho, S.C.; Euclydes, R.F.; Lana, R.P. e Queiroz, D.S. 2001. Cromo e indicadores internos na estimação do consumo de novilhos mestiços, suplementados, a pasto. Rev Bras Zootecn, 30: 1600-1609.

Detmann, E.; Paulino, M.F.; Zervoudakis, J.T.; Cecon, P.R.; Valadares Filho, S.C.; Gonçalves, L.C.; Cabral, L.S. e Melo, A.J.N. 2004. Níveis de proteína bruta em suplementos múltiplos para terminação de novilhos mestiços em pastejo durante a época seca: desempenho produtivo e características de carcaça. Rev Bras Zootecn, 33: 169-180.

Detmann, E. and Valadares Filho, S.C. 2010. On the estimation of non-fibrous carbohydrates in feeds and diets. Arq Bras Med Vet Zoo, 62: 980-984

Fernandes, H.J.; Paulino, M.F.; Detmann, E.; Valadares Filho, S.C.; Silva, A.G.; Porto, M.O.; Rocha, A.A. e Biancardi, G.F. 2012. Avaliação nutricional, durante a amamentação, de tourinhos em pastejo recebendo suplementação proteica da amamentação à terminação. Rev Bras Zootecn, 41: 374-383.

Henriques, L.T.; Valadares Filho, S.C.; Fonseca, M.A.; Paulino, P.V.R.; Detmann, E. e Valadares, R.F.D. 2011. Avaliação de modelos não-lineares e da relação doconsumo voluntário de vacas primíparas e de bezerros coma curva de lactação de vacas Nelore. Rev Bras Zootecn, 40: 1287-1295.

Lazzarini, I.; Detmann, E.; Sampaio, C.B.; Paulino, M.F.; Valadares Filho, S.C.; Souza, M.A. and Oliveira, F.A. 2009. Intake and digestibility in cattle fed low-quality tropical forage and supplemented with nitrogenous compounds. Rev Bras Zootecn, 38: 2021-2030.

Licitra, G.; Hernandez, T.M. and Van Soest, P.J. 1996. Standardization of procedures for nitrogen fractionation of ruminant feeds. Anim Feed Sci Tech, 57: 347-358.

Mertens, D.R. 2002. Gravimetric determination of amylase-treated neutral detergent fiber in feeds with refluxing in beaker or crucibles: collaborative study. J AOAC Int, 85: 1217-1240.

Nogueira, E.; Morais, M.G.; Andrade, V.J.; Rocha, E.D.S.; Silva, A.S. e Brito, A.T. 2006. Efeito do creepfeeding sobre o desempenho de bezerros e a eficiência reprodutiva de primíparas Nelore, em pastejo. Arq Bras Med Vet Zoo, 58: 607-613.
Paulino, M.F.; Figueiredo, D.M.; Moraes, E.H.B.K.; Porto, M.O.; Sales, M.F.L.; Acedo, T.S.; Villela, S.D.J. e Valadares Filho, S.C. 2004. Suplementação de bovinos em pastagens: uma visão sistêmica. Simpósio de produção de gado de corte, 4. Anais. Universidade Federal de Viçosa. Viçosa, MG. pp. 93-144.

Paulino, M.F.; Moraes, E.H.B.K.; Zervoudakis, J.T.; Alexandrino, E. e Figueiredo D.M. 2006. Terminação de novilhos mestiços leiteiros sob pastejo, no período das águas, recebendo suplementação com soja. Rev Bras Zootecn, 35: 154-158.

Porto, M.O.; Paulino, M.F.; Valadares Filho, S.C.; Detmann, E.; Sales, M.F.L. e Couto, V.R.M. 2009. Fontes de energia em suplementos múltiplos para bezerros Nelore em creep-feeding: desempenho produtivo, consumo e digestibilidade dos nutrientes. Rev Bras Zootecn, 38: 1329-1339.

Sampaio, C.B.; Detmann, E.; Lazzarini, I.; Souza, M.A.; Paulino, M.F. and Valadares Filho, S.C. 2009. Rumen dynamics of neutral detergent fiber in cattle fed low-quality tropical forage and supplemented with nitrogenous compounds. Rev Bras Zootecn, 38: 560-569.

Sampaio, A.A.M.; Fernandes, A.R.M.; Henrique, W.; Ribeiro, G.M.; Oliveira, E.A. e Silva, T.M. 2010. Efeito da suplementação sobre a digestibilidade dos nutrientes e desempenho de bezerros lactentes mantidos em pastagens de Brachiaria brizantha. Acta Sci Anim Sci, 32: 63-68.

Titgemeyer, E.C.; Armendariz C.K.; Bindel, D.J.; Greenwood, R.H. and Löest, C.A. 2001. Evaluation of titanium dioxide as a digestibility marker for cattle. J Anim Sci, 79: 1059-1063.

Valadares, R.F.D.; Broderick, G.A.; Valadares Filho, S.C. and Clayton, M.K. 1999. Effect of replacing alfalfa silage with high moisture corn on ruminal protein synthesis estimated from excretion of total purine derivatives. J Dairy Sci, 82: 2686-2696.

Valadares, R.F.D.; Goncalves, L.C.; Rodriguez, N.M.; Valadares Filho, S.C. e Sampaio, I.B.M. 1997. Níveis de proteína em dietas de bovino. 4. Concentrações de amônia ruminal e uréia plasmática e excreções de uréia e creatinina. Rev Bras Zootecn, 26: 1270-1278.

Valente, T.N.P.; Detmann, E.; Queiroz, A.C.; Valadares Filho, S.C.; Gomes, D.I. and Figueiras, J.F. 2011 . Evaluation of rumen degradation profiles of forages using bags made from different textiles. Rev Bras Zootecn, 40: 2565-2573.

Van Soest, P.J. and Robertson, J.B. 1985. Analysis of forages and fibrous foods. Cornell University. Ithaca. $202 \mathrm{pp}$.

Willians, C.H.; David, D.J. and lisma, O. 1962. The determination of chromic oxide in faeces samples by atomic absorption spectrophotometry. J Agr Sci, 59: 381-385. 